\title{
The $R_{b}$ Excess at LEP: Clue to New Physics at the TEVATRON? *
}

\author{
Ernest $\mathrm{Ma}^{1}$ and Daniel $\mathrm{Ng}^{2}$ \\ ${ }^{1}$ Department of Physics, University of California, \\ Riverside, California 92521, USA \\ ${ }^{2}$ TRIUMF, 4004 Wesbrook Mall, Vancouver, \\ British Columbia, Canada V6T $2 A 3$
}

\begin{abstract}
If the $R_{b}$ excess at LEP is real, then any explanation in terms of renormalizable loop corrections leads to important new decay modes of the $t$ quark and suppresses the $t \rightarrow b W$ branching ratio. In the two-Higgs-doublet model, the branching ratio of $Z \rightarrow b \bar{b}+$ a light boson which decays itself predominantly into $b \bar{b}$ is at least of order $10^{-4}$.
\end{abstract}

\section{INTRODUCTION}

The awesome statistics of the four LEP collaborations have pinned down with great precision a host of measurable parameters in the standard model [1]. The only quantity that shows a possible significant discrepancy with the theoretical prediction of the standard model is $R_{b}$ which is defined as

$$
R_{b} \equiv \frac{\Gamma(Z \rightarrow b \bar{b})}{\Gamma(Z \rightarrow \text { hadrons })}
$$

Using $m_{t}=175 \mathrm{GeV}$ and $m_{H}=300 \mathrm{GeV}$, the standard model predicts that $R_{b}=0.2158$, whereas LEP obtained $R_{b}=0.2202 \pm 0.0020$ if the similarly defined $R_{c}$ is assumed to be independent. If the latter is fixed at its standardmodel value, then $R_{b}=0.2192 \pm 0.0018$. In either case, the excess is about $2 \% \pm 1 \%$. If this is taken seriously, physics beyond the standard model is indicated.

\section{TWO HIGGS DOUBLETS}

The simplest extension of the standard model is to have two Higgs doublets instead of just one. The relevance of this model to $R_{b}$ was studied in detail already a few years ago [2]. To establish notation, let the two Higgs doublets be given by

$$
\Phi_{i}=\left(\begin{array}{c}
\phi_{i}^{+} \\
\phi_{i}^{0}
\end{array}\right)=\left[\begin{array}{c}
\phi_{i}^{+} \\
2^{-1 / 2}\left(v_{i}+\eta_{i}+i \chi_{i}\right)
\end{array}\right]
$$

Let $\tan \beta \equiv v_{2} / v_{1}$, then

$$
\begin{aligned}
h^{+} & =\phi_{1}^{+} \cos \beta-\phi_{2}^{+} \sin \beta, \\
h_{1} & =\eta_{1} \sin \alpha+\eta_{2} \cos \alpha, \\
h_{2} & =\eta_{1} \cos \alpha-\eta_{2} \sin \alpha, \\
A & =\chi_{1} \cos \beta-\chi_{2} \sin \beta .
\end{aligned}
$$

Note that the $\bar{b} b A$ and $\bar{t} b h^{+}$couplings involve the ratio $m_{b} \tan \beta / M_{W}$, hence they could be important for large values of $\tan \beta$. It was shown [2] that for $\tan \beta=70 \simeq 2 m_{t} / m_{b}$, the $R_{b}$ excess peaks at about $4 \%$ near $m_{A}=m_{h_{1}} \simeq 40 \mathrm{GeV}$ for $\alpha=0$. However, since $Z \rightarrow A h_{1}$ is not observed, $m_{A}+m_{h_{1}}>M_{Z}$ is a necessary constraint. We show in Fig. 1 the contours in the $m_{h_{1}}-m_{A}$ plane for 3 values of $R_{b}$. It is clear that relatively light scalar bosons are required if the $R_{b}$ excess is to be explained.

UCRHEP-T144

TRI-PP-95-16

April 1995

*in the proceedings of the International Symposium on Vector Boson Self-Interactions, UCLA, February 1995 


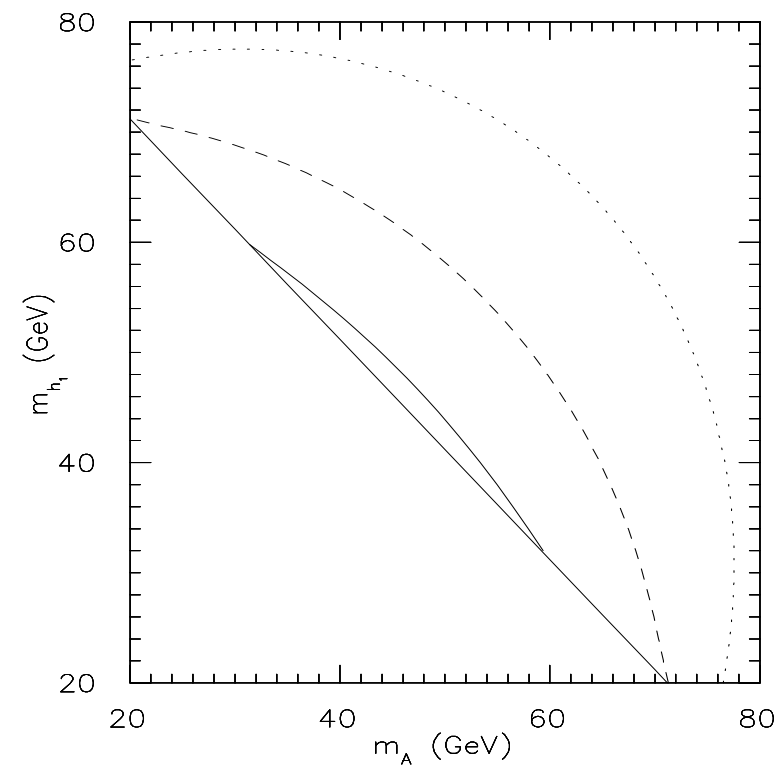

FIG. 1. $R_{b}=0.2192$ (solid), 0.2174 (dashed) and 0.2164 (dotted) contours in the $m_{h_{1}}-m_{A}$ plane for $\alpha=0$ and tan $\beta=70$. The straight line corresponds to $m_{A}+m_{h_{1}}=M_{Z}$. We have also assumed $m_{h^{ \pm}}=m_{h_{2}}=175 \mathrm{GeV}$.

For $A\left(h_{1}\right)$ lighter than $M_{Z}$ and having an enhanced coupling to $\bar{b} b$, the decay $Z \rightarrow b \bar{b}+A\left(h_{1}\right)$ becomes nonnegligible [3]. As an illustration, we show in Fig. 2 the branching ratios of these two decays as functions of $m_{A}$ with the constraint $m_{A}+m_{h_{1}}=M_{Z}+10 \mathrm{GeV}$ so that a reasonable fit to the $R_{b}$ excess is obtained. It is seen that the sum of these two branching ratios is at least of order $10^{-4}$. Once produced, $A$ or $h_{1}$ decays predominantly into $b \bar{b}$ as well. Hence this scenario for explaining $R_{b}$ can be tested at LEP if the sensitivity for identifying a $b \bar{b}$ pair as coming from $A$ or $h_{1}$ in $b \bar{b} b \bar{b}$ final states can be pushed down below $10^{-4}$.

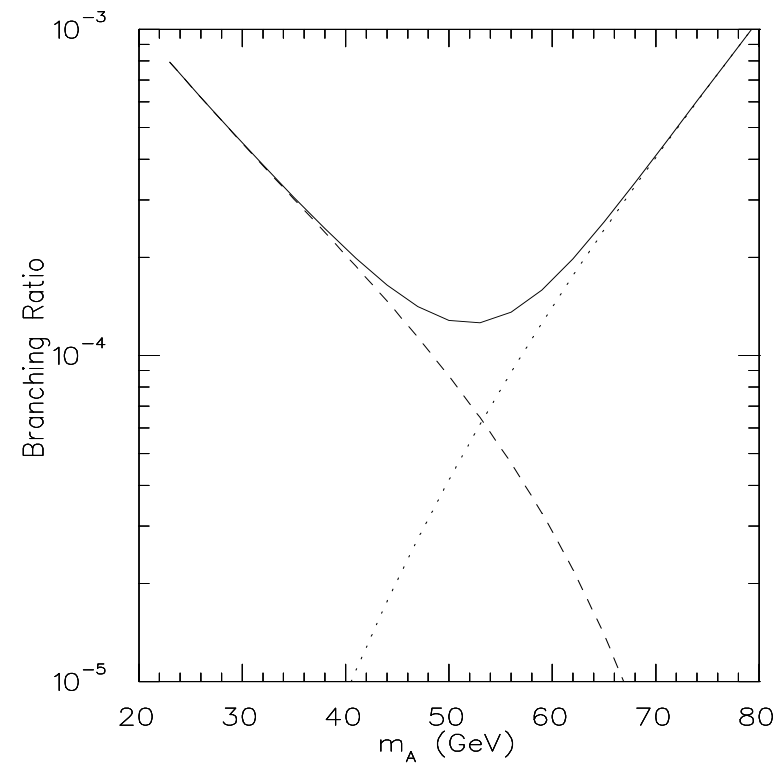

FIG. 2. The branching ratios, $\operatorname{Br}(Z \rightarrow b \bar{b} A)$ (dashed) and $\operatorname{Br}\left(Z \rightarrow b \bar{b} h_{1}\right)$ (dotted) and their sum (solid), as functions of $m_{A}$ where we take $m_{A}=m_{h_{1}}=M_{Z}+10 \mathrm{GeV}, \tan \beta=70, \alpha=0$, and $m_{h^{ \pm}}=m_{h_{2}}=175 \mathrm{GeV}$. 


\section{MINIMAL SUPERSYMMETRIC STANDARD MODEL}

In the Minimal Supersymmetric Standard Model (MSSM), there are two Higgs doublets, but their parameters are further constrained, hence the allowed region in the $m_{h_{1}}-m_{A}$ plane which gives a large enough $R_{b}$ is further reduced by the experimental nonobservation of MSSM signals at LEP [4.

There is of course another possible contribution to $R_{b}$ in the MSSM: the $Z \rightarrow b_{L} b_{L}$ vertex may be enhanced by the supersymmetric coupling of $b_{L}$ to a scalar top quark and a chargino [5]. In this case, both of the new particles must again be light, but now $Z$ cannot decay into just one of these particles because of the assumed conservation of $R$ parity, hence no further constraint is obtainable at LEP.

\section{NECESSARY TOP DECAYS}

Since $b_{L}$ is involved in any enhanced coupling to light particles in explaining the $R_{b}$ excess, its doublet partner $t_{L}$ must necessarily have the same enhanced coupling to related particles. In the two-Higgs-doublet case, we must have an enhanced $\bar{t} b h^{+}$coupling. Therefore, unless $m_{h^{+}}>m_{t}-m_{b}$, the branching ratio of $t \rightarrow b h^{+}$will dominate over all others. In particular, the standard $t \rightarrow b W$ branching ratio will be seriously degraded. We show in Fig. 3 the branching ratio $\operatorname{Br}(t \rightarrow b W)$ as a function of $m_{h^{+}}$. Large values of $m_{h^{+}}$are disfavored in this scenario because the splitting with $A$ and $h_{1}$ would result in a large contribution to the $\rho$ parameter [6]. This poses a problem for top production at the TEVATRON because the number of observed top events is consistent with the assumption that top decays into $b W 100 \%$ of the time. If that is not so, then top production must be enhanced by a large factor beyond that of the standard model. The two-Higgs-doublet model itself certainly does not provide for any such mechanism.

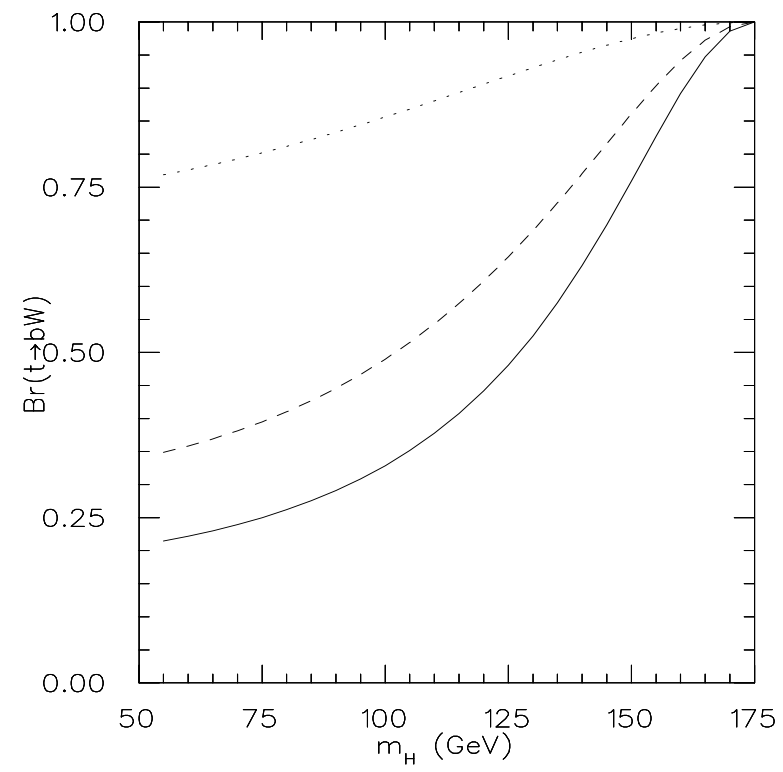

FIG. 3. The branching ratio $B r(t \rightarrow b W)$ as a function of $m_{h}^{+}$for $\tan \beta=70$ (solid), 50 (dashed), and 20 (dotted).

In the MSSM, if the $R_{b}$ excess is attributed to a light scalar top quark and a light chargino, then we should look at the latter's doublet partner which is in general a linear combination of neutralino mass eigenstates. At least one of these, i.e. the Lightest Supersymmetric Particle (LSP), will be light enough to allow the top quark to decay into it and the scalar top. The $\rho$ parameter also serves to disfavor large neutralino masses in this scenario. Hence the $t \rightarrow b W$ branching ratio is again seriously degraded. Turning the argument around, this means that for every observed top event, there must be several others which correspond to the production of supersymmetric particles. If the $R_{b}$ excess is really due to supersymmetry, top decay is the place to discover it! 


\section{CONCLUSION}

If the $R_{b}$ excess at LEP is real and we want to explain it in terms of renormalizable loop corrections, then light particles are unavoidable. However, these light particles may be produced also in $Z$ decay such as in the two-Higgsdoublet case, where $Z \rightarrow b \bar{b}+A$ or $h_{1}$ is at least of order $10^{-4}$ in branching ratio. More importantly, there is necessarily a corresponding top decay into one of these light particles (such as the scalar top in the MSSM) and the other particle's doublet partner (the neutralino), which seriously degrades the $t \rightarrow b W$ branching ratio. Unless there is accompanying new physics which enhances the top production by a large factor at the TEVATRON, this generic explanation of the $R_{b}$ excess in terms of light particles does not appear to be viable.

\section{ACKNOWLEDGEMENT}

The work of E.M. was supported in part by the U.S. Department of Energy under Contract No. DE-AT0387ER40327. The work of D.N. was supported by the Natural Sciences and Engineering Research Council of Canada.

[1] The LEP Collaborations: ALEPH, DELPHI, L3, OPAL, and the LEP Electroweak Working Group, CERN Report No. CERN/PPE/94-187 (25 November 1994).

[2] A. Denner, R. J. Guth, W. Hollik, and J. H. Kühn, Z. Phys. C51, 695 (1991).

[3] A. Djouadi, P. M. Zerwas, and J. Zunft, Phys. Lett. B259, 175 (1991).

[4] See for example A. Sopczak, CERN Report No. CERN/PPE/94-73 (9 May 1994).

[5] G. Altarelli, R. Barbieri, and F. Caravaglios, Phys. Lett. B324, 357 (1993); J. D. Wells, C. Kolda, and G. L. Kane, Univ. of Michigan Report No. UM-TH-94-23 (July 1994); D. Garcia, R. A. Jimenez, and J. Sola, Univ. of Barcelona Reports Nos. UAB-FT-343,344 (September 1994).

[6] A. K. Grant, Phys. Rev. D 51, 207 (1995). 